\title{
Development of Graphene Nanoplatelet-Reinforced AZ91 Magnesium Alloy by Solidification Processing
}

\author{
Sinan Kandemir
}

(Submitted January 25, 2018; in revised form April 19, 2018; published online April 27, 2018)

\begin{abstract}
It is a challenging task to effectively incorporate graphene nanoplatelets (GNPs) which have recently emerged as potential reinforcement for strengthening metals into magnesium-based matrices by conventional solidification processes due to their large surface areas and poor wettability. A solidification processing which combines mechanical stirring and ultrasonic dispersion of reinforcements in liquid matrix was employed to develop AZ91 magnesium alloy matrix composites reinforced with 0.25 and 0.5 wt.\% GNPs. The microstructural studies conducted with scanning and transmission electron microscopes revealed that fairly uniform distribution and dispersion of GNPs through the matrix were achieved due to effective combination of mechanical and ultrasonic stirring. The GNPs embedded into the magnesium matrix led to significant enhancement in the hardness, tensile strength and ductility of the composites compared to those of unreinforced AZ91 alloy. The strength enhancement was predominantly attributed to the grain refinement by the GNP addition and dislocation generation strengthening due to the coefficient of thermal expansion mismatch between the matrix and reinforcement. The improved ductility was attributed to the refinement of $\beta$ eutectics by transforming from lamellar to the divorced eutectics due to the GNP additions. In addition, the strengthening efficiency of the composite with $0.25 \mathrm{wt} \%$ GNP was found to be higher than those of the composite with $0.5 \mathrm{wt} \%$ GNP as the agglomeration tendency of GNPs is increased with increasing GNP content. These results were compared with those of the GNP-reinforced magnesium composites reported in the literature, indicating the potential of the process introduced in this study in terms of fabricating light and high-performance metal matrix composites.
\end{abstract}

Keywords graphene nanoplatelets, magnesium alloys, mechanical properties, metal matrix composites, microstructure

\section{Introduction}

Magnesium (Mg), which is one of the lightest structural metals with a density about two-thirds that of aluminum (Al), has always been an attractive material particularly for the automotive industry to contribute energy efficiency (Ref 1, 2). However, magnesium and its alloys suffer from limited strength and ductility that is arisen from the limited number of slip systems in HCP crystal structure. Therefore, in order to broaden the range of engineering applications, it is required to focus on developing conventional $\mathrm{Mg}$ alloys and composites, in which the mechanical properties are tailored. In earlier research, $\mathrm{Mg}$ was strengthened with micron-sized ceramic reinforcements such as $\mathrm{Al}_{2} \mathrm{O}_{3}, \mathrm{SiC}, \mathrm{TiC}$ and $\mathrm{TiB}_{2}$, but the main disadvantage associated with these composites is poor ductility due to high volume fraction and brittle nature of the reinforcements (Ref 3, 4). In addition, it has been shown that the incorporation of nanosized reinforcements, e.g., nanoparticles, into $\mathrm{Mg}$ and its alloys can significantly improve the strength without compromising the ductility (Ref 5-7). During the last decade, carbon nanotubes (CNTs) have been considered alternative reinforcement to ceramic nanoparticles for strengthening $\mathrm{Mg}$ and its

Sinan Kandemir, Department of Mechanical Engineering, Izmir Institute of Technology, 35430 Urla, Izmir, Turkey. Contact e-mail: sinankandemir@iyte.edu.tr. alloys owing to their high mechanical strength as well as low density (Ref 8-10). However, the main challenge is to obtain uniform dispersion of CNTs into metals due to their high agglomeration tendency caused by strong van der Waals forces (Ref 11). This issue may lead to poor mechanical properties in $\mathrm{Mg} / \mathrm{CNT}$ composites. Another challenge is the preservation of integrity of CNTs during the processes that require mechanical milling. Therefore, CNTs do not seem the most suitable candidate for reinforcing $\mathrm{Mg}$-based matrices.

Graphene, a two-dimensional material comprising of a single layer of carbon atoms, has attracted tremendous attention due to its unique mechanical and physical properties (Ref 12). Recently, industrially produced graphene nanoplatelets (GNPs) that consist of a few graphene layers with a thickness of $<100 \mathrm{~nm}$ have emerged as potential reinforcement for strengthening metals, i.e., $\mathrm{Al}, \mathrm{Cu}$ and $\mathrm{Mg}$ (Ref 13-15). To date, several attempts were made to fabricate GNP-reinforced Mg-based matrix composites with powder metallurgy and solidification processing routes (Ref 15-17). Solidification processing is considered to be more suitable in terms of reducing the cost of composite production and hence mass production. On the other hand, solidification processing could bring additional issues over the agglomeration tendency of GNPs due to their large surface area in the fabrication of $\mathrm{Mg}$ / GNP composites. These include the poor wetting of GNPs by molten $\mathrm{Mg}$ and pushing GNPs ahead of the solidification front that results in cluster formation at grain boundaries.

There are few systematic investigations regarding GNPreinforced $\mathrm{Mg}$ matrix composites fabricated by solidificationbased processes in the literature. Chen et al. (Ref 15) developed a method combining liquid-state ultrasonic processing and solid-state friction stirring (post-processing) to incorporate 
GNPs into the Mg matrix and showed impressive enhancement in the hardness of composite. Rashad et al. (Ref 18) used solidification processing to reinforce Mg-6Zn alloy with GNPs, but the uniform dispersion and distribution of GNPs throughout the matrix were not documented. Very recently, Xiang et al. (Ref 19) have reinforced pure $\mathrm{Mg}$ with GNPs via the disintegrated melt deposition method, which is a combination of spray processing (gas disintegration) and traditional casting. The experimental results suggested that the overall strength and ductility of composites were improved with the homogeneous distribution and dispersion of GNPs compared to those of the matrix. Despite the substantial enhancement shown in the previous studies, further research is required for improvement in the incorporation and dispersion rate of GNPs into liquid $\mathrm{Mg}$ matrices in order to maximize the potential of GNPs as reinforcements. Also, to the best of our knowledge, open literature sources so far suggested that no study was conducted to incorporate GNPs into AZ91 alloy which is used in the majority of all magnesium cast components in industry.

Therefore, the aim of the present work is to fabricate GNPreinforced AZ91 alloys using liquid-state ultrasonic processing, in which reinforcements are dispersed under ultrasonic cavitation zone generated by a high-power ultrasonic probe, and to investigate their microstructure and mechanical properties. The method described here for feeding the reinforcement into the matrix involves firstly simple ball milling of GNPs with $\mathrm{Mg}$ powders for the deagglomeration of GNPs, and forming pellets from the mixture of GNPs and Mg powders, and finally melting these pellets in the alloy.

\section{Experimental Procedure}

\subsection{Materials}

For this study, AZ91 (Mg-9Al-0.8Zn-0.2Mn) magnesium cast alloy and commercially available GNPs having a thickness of 50-100 nm with an average $x, y$ dimension of $5 \mu \mathrm{m}$ were used as the matrix and reinforcement, respectively. The scanning electron microscopy (SEM) images of the as-received GNPs are given in Fig. 1. For the deagglomeration of GNPs by ball milling prior to the composite fabrication, $\mathrm{Mg}$ and $\mathrm{Al}$ powders with $99 \%$ purity were used. The main reason to add $\mathrm{Al}$ powders into the composite is to improve the wetting between GNPs and Mg matrix, since graphene has better wettability by $\mathrm{Al}$ compared to $\mathrm{Mg}$ ( $\mathrm{Ref} 20)$. In addition, $\mathrm{Al}$ is compatible with the alloy as it exists in the alloy composition. Figure 2 shows the SEM micrographs of as-received $\mathrm{Mg}$ (Fig. 2a) and $\mathrm{Al}$ (Fig. 2b) powders with average sizes of $<75 \mu \mathrm{m}$.

\subsection{Fabrication of the Composites}

It is known that direct introduction of nanosized reinforcements into liquid alloys may lead to severe reinforcement agglomeration and flotation on the melt surface. In order to overcome this issue, the pellets, in which the GNPs are encapsulated with $\mathrm{Mg}$ and $\mathrm{Al}$ powders, were prepared to be fed into molten metals. For the preparation of pellets, appropriate amount of $\mathrm{Mg}$ and $\mathrm{Al}$ powders was first mixed with GNPs in stainless steel vials of a planetary ball mill (Retsch PM 200) for $2 \mathrm{~h}$ at $350 \mathrm{rpm}$. $\mathrm{Mg} / \mathrm{Al}$ weight ratio in the pellet was to be $25: 3$ to not change the chemical composition of alloy. The required weight of GNPs was added into the vials for the fabrication of composites with different GNP contents. For example, $12.5 \mathrm{~g} \mathrm{Mg}$ and $1.5 \mathrm{~g} \mathrm{Al}$ powders were ball-milled with $0.75 \mathrm{~g}$ GNPs for AZ91/0.50 wt.\% GNP composite. The stainless steel balls-to-total powders weight ratio was $4: 1$, and diameter of the balls was $10 \mathrm{~mm}$. The milling operation was interrupted every $15 \mathrm{~min}$ for $5 \mathrm{~min}$ to allow cooling of $\mathrm{Mg}$ powders that can easily burn in air. The ball-milled powders and GNPs were then pressed under $250 \mathrm{MPa}$ to form pellets $30 \mathrm{~mm}$ in diameter.

The schematic experimental setup for the fabrication of GNP-reinforced Mg matrix composites, which consists of an electric resistance furnace, a mechanical stirrer (IKA, RW 20) and an ultrasonic unit (Q700 sonicator, Qsonica, LLC), is shown in Fig. 3. The ultrasonic unit is capable of generating $20 \mathrm{kHz}$ ultrasonic waves with a maximum $700 \mathrm{~W}$ power output. The AZ91 alloy was melted in a graphite crucible with a total melt volume of $130 \mathrm{~g}$ at $675{ }^{\circ} \mathrm{C}$. At this temperature, the molten alloy was mechanically stirred with a graphite stirrer at $1000 \mathrm{rpm}$. The pellets containing the GNPs were introduced into the melt during mechanical stirring, and the composite was stirred with a total processing time of $15 \mathrm{~min}$ (Fig. 3a). It is a fact that the temperature of alloy was dropped into the semisolid region $\left(575-590{ }^{\circ} \mathrm{C}\right)$, while the pellets were being melted. It was envisioned that the semisolid-state stirring could facilitate the engulfment of GNPs into the matrix by reducing their buoyancy. After the stirrer was removed, the melt was reheated to $700{ }^{\circ} \mathrm{C}$ and the $12.7-\mathrm{mm}$-diameter titanium alloy Ti-6Al-4V ultrasonic probe that is a part of the ultrasonic unit was submerged into the molten alloy. The melt was ultrasonically processed for $15 \mathrm{~min}$ at $675^{\circ} \mathrm{C}$ with a peak-to-peak amplitude of about $45 \mu \mathrm{m}$ (Fig. 3b). After the ultrasonic processing, the molten composite was reheated to $700{ }^{\circ} \mathrm{C}$ and
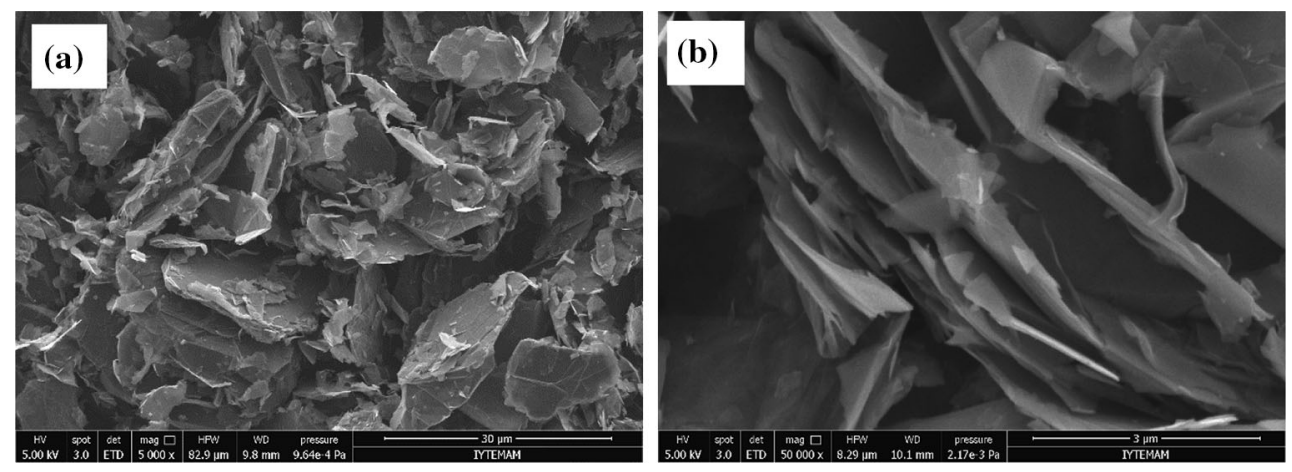

Fig. 1 SEM micrographs of as-received GNPs at magnifications of (a) $\times 5000$ and (b) $\times 50,000$ 

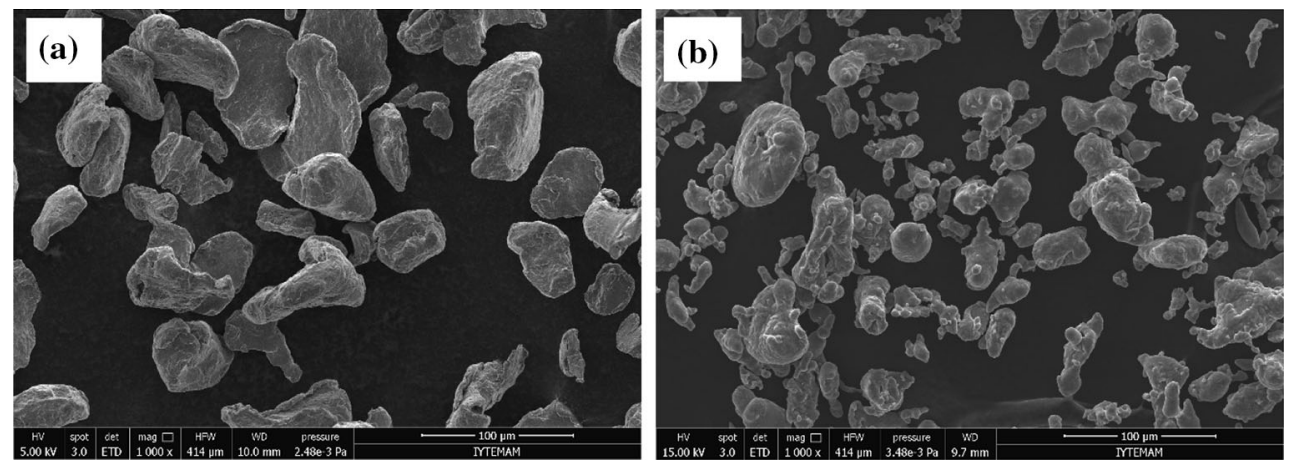

Fig. 2 SEM micrographs of as-received (a) Mg and (b) Al powders used for the deagglomeration of GNPs by ball milling
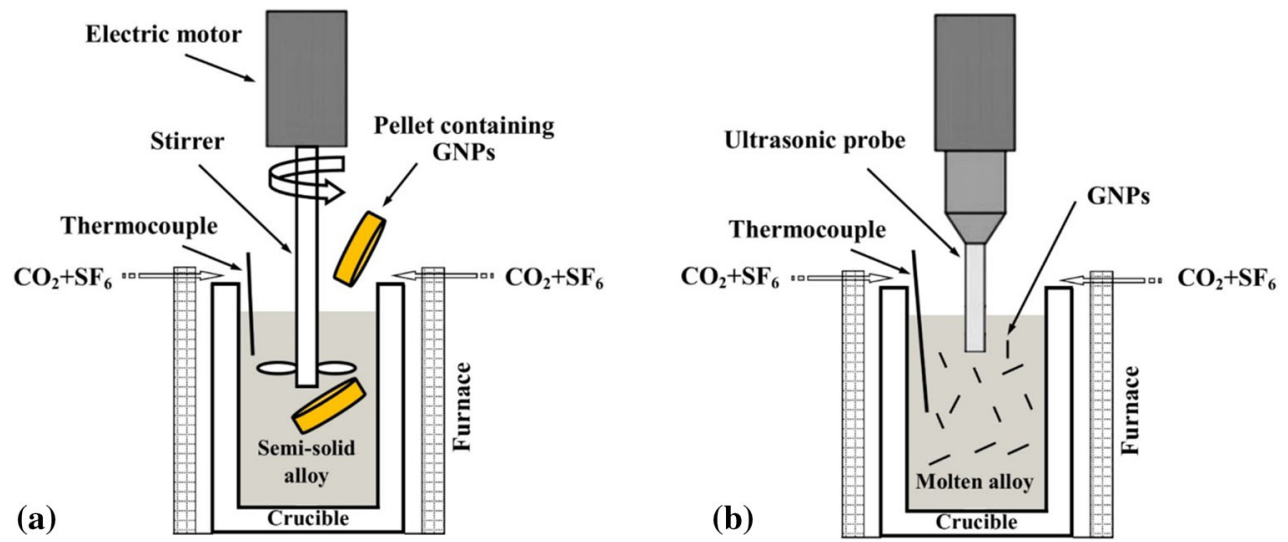

Fig. 3 Schematic experimental setup for the fabrication of GNP-reinforced AZ91 alloy: (a) mechanical stirring of alloy with the introduction of pellets and (b) ultrasonic dispersion of GNPs in the alloy

then cast into a steel mold preheated to $350{ }^{\circ} \mathrm{C}$ to prepare tensile test specimens. The entire process was conducted under a protective atmosphere of $99 \% \mathrm{CO}_{2}+1 \% \mathrm{SF}_{6}$. The $\mathrm{Mg}$ matrix composites were produced with approximately 0.25 and 0.5 wt.\% GNP. For comparison, unreinforced AZ91 reference castings were also produced using the pellets without GNPs under similar processing and solidification parameters.

\subsection{Microstructural Characterization and Mechanical Testing}

For the microstructural characterization, the samples that were conventionally ground and polished down to $0.5-\mu \mathrm{m}$ finish with alumina-based suspension were examined by means of a SEM (FEI Quanta FEG 250) equipped with an energydispersive $\mathrm{x}$-ray spectroscopy (EDX) system. A transmission electron microscope (TEM, FEI Tecnai F30) operating at $200 \mathrm{kV}$ was also employed in order to investigate the bonding between the matrix and GNPs. The TEM samples were prepared by cutting thin slices from the composite specimens with an ultramicrotome device (Leica EM UC6). These electron transparent slices with a thickness of less than $100 \mathrm{~nm}$ were placed on a copper TEM grid and examined under the microscope. The SEM and TEM samples were not subjected to etching in order to protect potential structures that may be observed between the matrix and reinforcement. However, some samples were etched with acetic picral for the determination of average grain sizes. The average grain sizes of $\alpha-\mathrm{Mg}$ grains for all samples were measured based on the linear intercept method, as described in ASTM E112 standards.

The Vickers hardness tests were conducted for the macrohardness testing of specimens with a load of $5 \mathrm{kgf}$ for $10 \mathrm{~s}$. At least ten measurements were taken each specimen and the average values were reported. For tensile testing, the dog-boneshaped specimens with a gage length of $20 \mathrm{~mm}$ and a gage section diameter of $6 \mathrm{~mm}$ were machined from the cast reference and GNP-reinforced AZ91 alloys based on ASTM E 8/E 8 M-08 standards. The tensile properties were determined using a universal tensile testing machine with $1 \mathrm{~mm} \times$ $\min ^{-1}$ crosshead speed at room temperature. Three casts were made for the reference alloy and composite separately. At least three tensile specimens from each cast were tested and the average values were presented.

\section{Results and Discussion}

\subsection{Microstructures}

SEM analysis was carried out for a cross section of the pellets in order to document the dispersion of GNPs through $\mathrm{Mg}$ and $\mathrm{Al}$ powders prior to their incorporation into the AZ91 matrix, as shown in Fig. 4. The EDX elemental mapping, in which $\mathrm{C}$ map potentially corresponds to the GNPs and their clusters, for a selected area in the microstructure of pellet, is given in Fig. 4(b). It is clearly seen from the analysis that the 
as-received GNPs were largely deagglomerated and encapsulated between the metal powders by high-energy ball milling. It is worth to note that single GNPs and relatively small-sized GNP clusters were unlikely to appear in the map due to spatial resolution limit of the EDX system. In addition, the weight of oxygen in the pellet was relatively low and this may suggest that the oxidation of powders during the ball milling process was not significant.

Figure 5 exhibits the SEM (BSE) micrographs of the AZ91 reference alloy without reinforcements and alloys reinforced with 0.25 and $0.5 \mathrm{wt} . \%$ GNP at relatively low magnification. The microstructure of AZ91 reference alloy (Fig. 5a) shows that $\beta$ phase which is the intermetallic phase appears as brighter regions surrounding the darker $\alpha-\mathrm{Mg}$ grains. As shown in Fig. 5(b) and (c), the incorporation of GNPs into AZ91 alloys led to refinement of primary $\alpha-\mathrm{Mg}$ grains and secondary $\beta$ phase. While the average grain size of the AZ91 alloy was $94 \pm 16 \mu \mathrm{m}$, those of the composites with 0.25 and 0.5 wt. $\%$ GNP were measured to be $72 \pm 13$ and $58 \pm 12 \mu \mathrm{m}$, respectively. This indicates that the grain size was reduced with increasing GNP contents. The significant reduction in grain sizes could be attributed to the grain boundary pinning effect of the GNPs.

Another important observation is the remarkable porosity formation with the addition of GNPs into the matrix (see Fig. 6c). The densities of samples were measured using Archimedes principle, and the differences between experimental and theoretical densities were found to be $2.5,3$ and $4 \%$ for the AZ91 alloys with $0,0.25$ and 0.5 wt.\% GNP, respectively.
This indicates that the porosity level increased with increasing GNP content in the AZ91 alloy. There could be two reasons for the increased porosity level due to the GNP addition. First is possible diminishing of melt fluidity by the GNP content, resulting in cast defects. It has been reported that graphene sheets may act as barriers to the diffusion of elements in alloys (Ref 15). The second reason may be that a significant amount of GNPs which was left as clusters possibly due to insufficient ultrasonic dispersion, particularly for relatively high GNP content, i.e., $0.5 \mathrm{wt} . \%$, and pushed to the grain boundaries, since these GNPs are likely to obstruct the diffusion during solidification, leading to high porosity in the composites (Ref 16).

For the investigation of morphological changes in the intermetallic phase by GNP addition, higher-magnification SEM images of the AZ91 alloy and its composite with 0.25 wt.\% GNP are given in Fig. 6. It is well established that $\alpha+\beta$ eutectic phase is also present along with $\alpha-\mathrm{Mg}$ and intermetallic $\beta$ in AZ91 alloys (Fig. 6a). The formation of $\beta$ and $\alpha+\beta$ eutectic phases is commonly considered to be due to the higher segregation tendency of $\mathrm{Zn}$ and the degree of constitutional undercooling ahead of the solidification front (Ref 21). It is evident from Fig. 6(b) that the lamellar eutectics were transformed into the fully divorced $\beta$ eutectics by the incorporation of GNPs into the matrix (Note that similar morphological traits to that of AZ91/0.25 wt.\% GNP composite were observed for the composite with 0.5 wt. $\%$ GNP). It has been suggested that alloying elements and solidification parameters could alter the eutectic morphology in monolithic

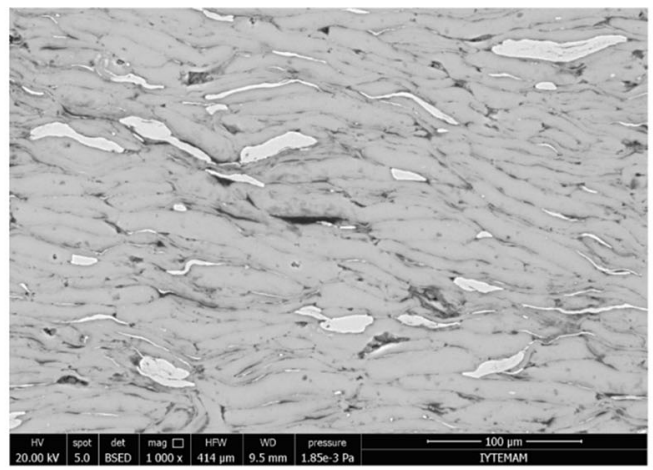

(a)
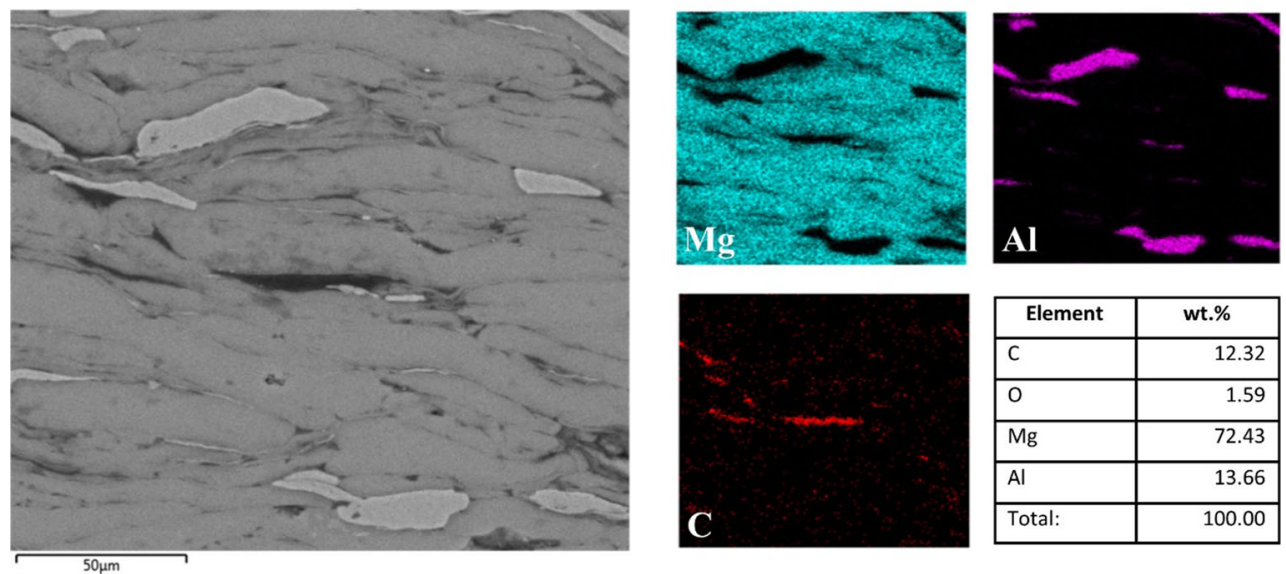

\begin{tabular}{|l|r|}
\hline \multicolumn{1}{|c|}{ Element } & \multicolumn{1}{c|}{ wt.\% } \\
\hline $\mathrm{C}$ & 12.32 \\
\hline $\mathrm{O}$ & 1.59 \\
\hline $\mathrm{Mg}$ & 72.43 \\
\hline $\mathrm{Al}$ & 13.66 \\
\hline Total: & 100.00 \\
\hline
\end{tabular}

(b)

Fig. 4 (a) Backscattered electron (BSE) image and (b) EDX mapping of an area selected in the microstructure of pellet containing the GNPs 

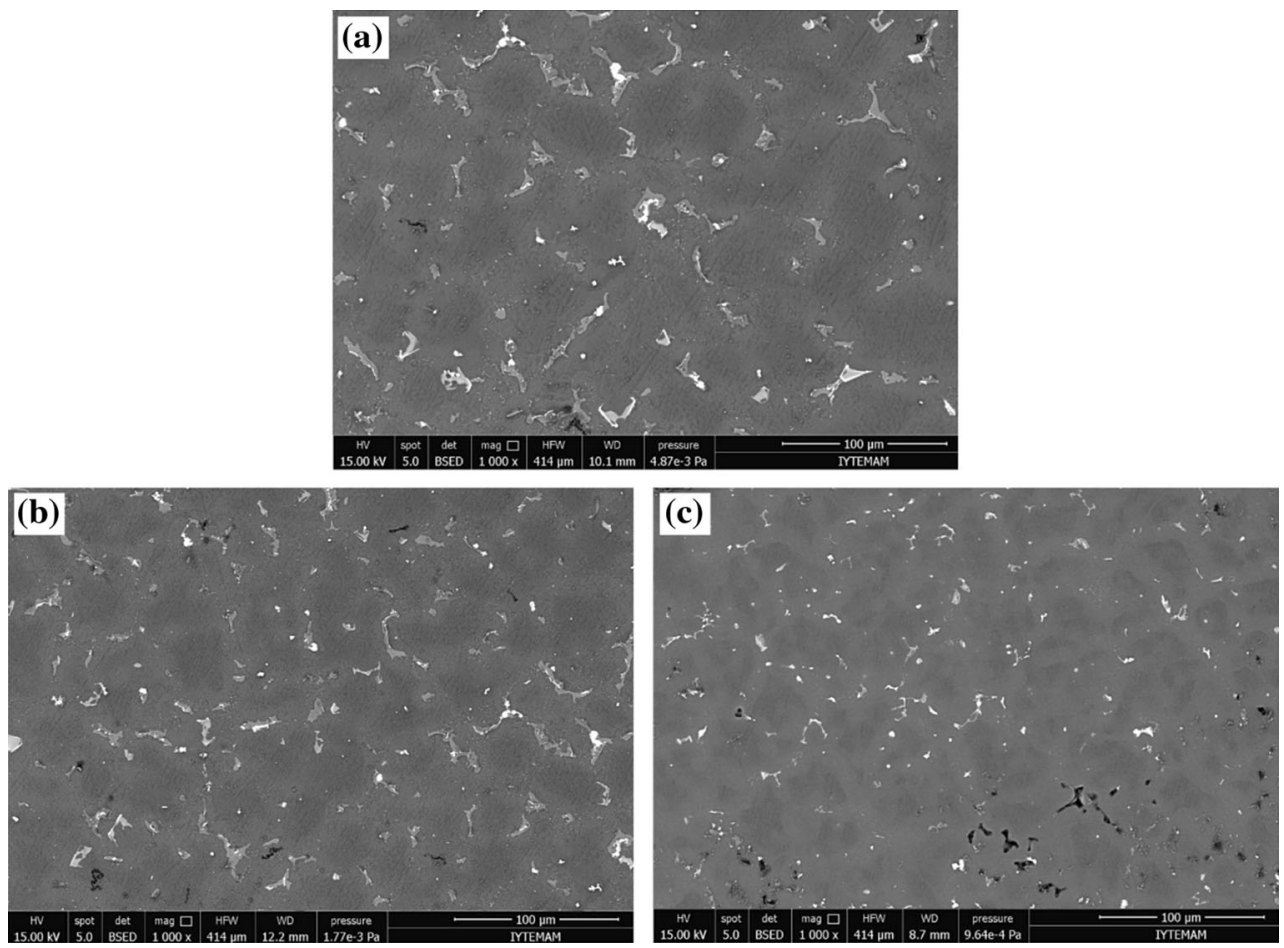

Fig. 5 BSE images of the AZ91 alloys with GNPs: (a) 0, (b) 0.25 and (c) 0.5 wt.\%
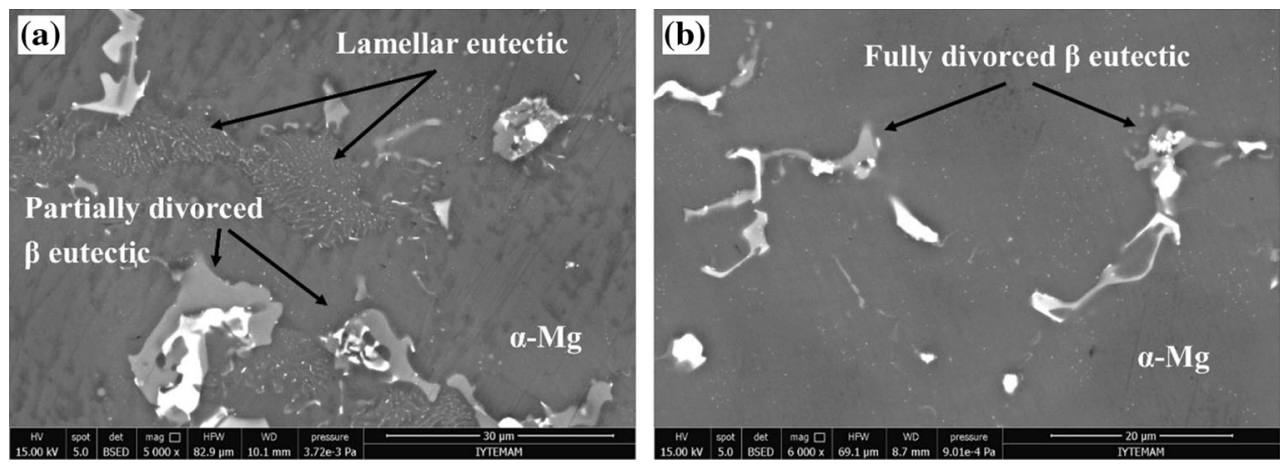

Fig. 6 Higher-magnification BSE images of (a) the AZ91 alloy and (b) the AZ91/0.25 wt.\% GNP composite (this figure has been modified in order to highlight the eutectic modification)

Mg alloys (Ref 22, 23). However, the addition of GNPs into AZ91 alloy in this study is unlikely to modify the phase composition, since no chemical reaction is expected to occur between the GNPs and matrix. In spite of the superb thermal conduction property of graphene, the GNPs incorporated into the matrix are not considered to significantly change the rate of solidification, hence the eutectic morphology due to a relatively low content of GNPs, i.e., 0.5 wt.\% max. Therefore, it could be suggested that the GNPs which act as diffusion barriers may have suppressed the diffusion of $\mathrm{Al}$ and $\mathrm{Mg}$ atoms during solidification and led to the refinement of $\beta$ eutectics by transforming from lamellar to the divorced eutectics (note that it is not possible to locate the GNPs under SEM at the magnification as indicated in Fig. 6). It seems in the open literature that no previous study has directly investigated the effect of carbonaceous nanofillers such as GNP and CNT on the morphological alteration of $\beta$ phase in AZ series alloys. Hence, it is thought that these preliminary results suggest further research in order to fully understand the detailed mechanism of morphological alterations of $\beta$ phase by GNP additions in AZ91 alloys.

Figure 7 compares the SEM images of AZ91/0.25 wt.\% GNP and AZ91/0.5 wt.\% GNP composites. It is seen that the GNPs with various contents were incorporated and relatively uniformly distributed into the alloy due to effective combination of mechanical and ultrasonic stirring. Large numbers of GNPs were found well dispersed throughout the matrix. However, few clusters of GNPs were observed in the AZ91/ 0.5 wt. $\%$ GNP composite, as indicated in Fig. 7(b). The presence of these clusters could be attributed to insufficient ball milling and/or ultrasonic energy for deagglomeration of increasing GNP content, i.e., $0.5 \mathrm{wt} . \%$, as the same processing parameters were used for the dispersion of 0.25 and $0.5 \mathrm{wt} . \%$ GNPs. Unlike micron-sized ceramic reinforcements, the GNPs also appeared to be incorporated in the $\alpha-\mathrm{Mg}$ grains, not only in the grain boundaries (Fig. $7 \mathrm{a}$ and $\mathrm{b}$ ). It has been previously 
reported for CNTs that solidification rate affects the distribution of reinforcements in a solidified metal matrix composite (Ref 24, 25). Thus, it can be inferred that GNPs could be pushed to the grain boundaries at a lower solidification rate and captured inside grains by the solidification front at a higher solidification rate. It could be therefore suggested that the solidification rate in the present study was relatively high to capture the dispersed GNPs into the $\alpha$-Mg grains. In addition, there is a noticeable difference between the as-received GNPs (Fig. 1) and the dispersed GNPs into the matrix (Fig. 7) in terms of their sizes. This can be ascribed to the fact that the as-received GNPs were broken into small-sized GNPs due to high-energy ball milling, which is expected to increase the dislocation density resulting in increased strength of composites. Furthermore, the EDX analysis was performed on a potential GNP that is singly dispersed into the AZ91/0.5 wt.\% GNP composite (Fig. 7b) in order to ascertain the presence of GNPs, as shown in Fig. 8. The distinct carbon peak in the analysis confirms the presence of carbonaceous fillers in the matrix.

The representative TEM micrographs of AZ91/0.25 wt.\% composite are shown in Fig. 9. The TEM images further confirmed that the GNPs with an interplanar distance of 0.34 were effectively dispersed and embedded into the $\mathrm{Mg}$ matrix with different orientations (Fig. 9b and c). It was also revealed that there is good interfacial bonding between the matrix and GNPs as no reaction products or no cavities were traced. This may suggest that the combination of proper feeding mechanism and ultrasonic dispersion of GNPs into matrix led to good bonding as well as good wetting of GNPs by molten Mg during the casting.

\subsection{Mechanical Properties}

The hardness test results and tensile properties of AZ91 reference alloy and its composites with 0.25 and $0.5 \mathrm{wt} \%$
GNPs are presented in Table 1. It is clear that the addition of GNPs into the AZ91 matrix led to an increase in the hardness values. 0.25 and $0.5 \mathrm{wt} \%$ GNP contents enhanced the hardness by approximately 8.3 and $15 \%$, respectively, compared to that of the reference alloy. This enhancement could be ascribed to the relatively uniform dispersion of harder GNPs that resist to plastic deformation induced by indentation and the grain refinement by GNPs.

It can be seen from Table 1 that the $0.2 \%$ proof stress (PS) and ultimate tensile strength (UTS) simultaneously increased with increasing GNP content. For the addition of 0.25 wt. $\%$, a $22 \%$ enhancement in PS and a $19 \%$ increase in UTS were observed. The PS and UTS were enhanced by 34 and $32 \%$, respectively, compared to those of the reference alloy when the GNP content was 0.5 wt. $\%$.

Several strengthening mechanisms could be expected to contribute to the strength enhancement in Mg-GNP composites. These are load bearing effects by the modulus change due to the addition of harder GNPs, grain size refinement by HallPetch effect, coefficient of thermal expansion (CTE) mismatch between the matrix and GNPs, and Orowan strengthening by the resistance of closely spaced GNPs to a moving dislocations. The load bearing effect is likely to be effective when a good bonding between reinforcement and matrix is achieved, and a large volume fraction of reinforcements is embedded into matrix (Ref 26, 27). As indicated earlier, good interfacial bonding was obtained between the GNPs and matrix (Fig. 9b and c). Besides, it has been proposed that the high specific surface area and wrinkled surface texture (Fig. 9a) of GNPs could promote the mechanical interlock resulting in efficient load transfer compared to spherical and fiber/whisker reinforcements (Ref 19). However, the GNP content in the composites is relatively low, i.e., max. $0.5 \mathrm{wt} . \%$, and the load bearing is therefore not considered to play a crucial role in strengthening the AZ91 alloy with the addition of GNPs.
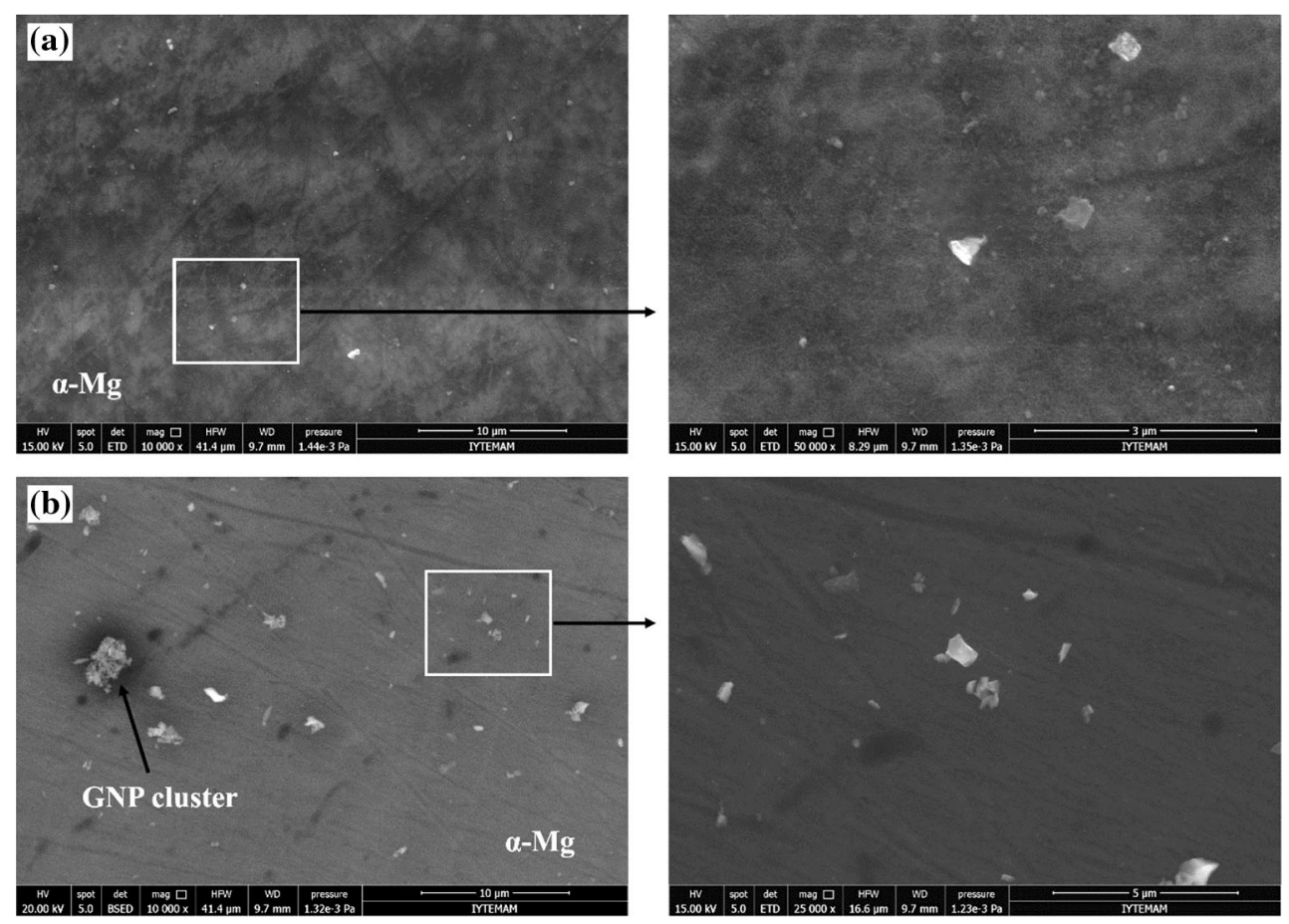

Fig. 7 SEM micrographs of (a) the AZ91/0.25 wt.\% GNP and (b) the AZ91/0.5 wt.\% GNP composites 

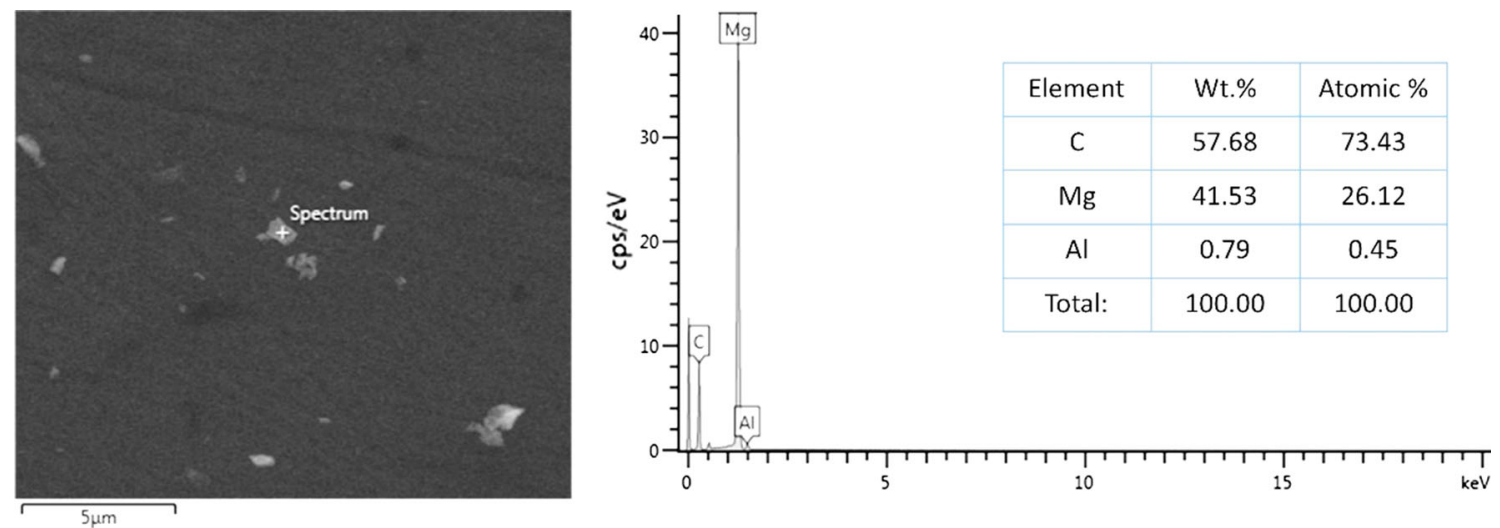

Fig. 8 EDX analysis for a potential GNP dispersed into the AZ91/0.5 wt.\% GNP composite
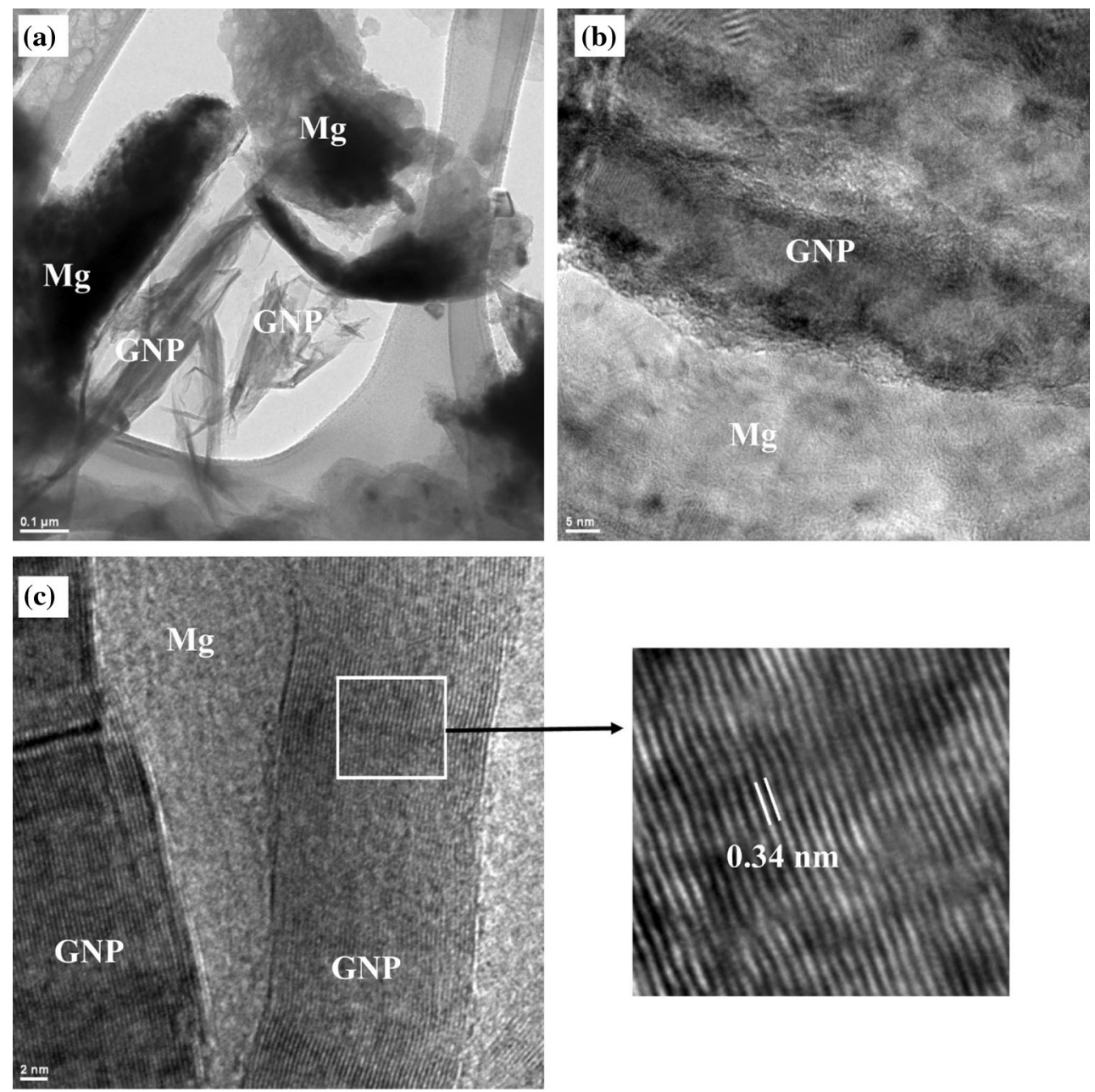

Fig. 9 Representative TEM images of the AZ91/0.25 wt.\% GNP composite showing (a) distribution of GNPs in the matrix, (b) and (c) single GNPs embedded into the matrix

Table 1 Mechanical properties of the AZ91 reference alloy and its GNP-reinforced composites

\begin{tabular}{lcccr}
\hline Specimen & Vickers hardness (HV) & $\mathbf{0 . 2 \%}$ Proof stress, MPa & Ultimate tensile strength, MPa & Elongation to fracture, \% \\
\hline AZ91 alloy & $60 \pm 0.5$ & $95 \pm 6$ & $144 \pm 8$ & $2 \pm 0.5$ \\
AZ91/0.25 wt.\% GNP & $65 \pm 1.5$ & $116 \pm 9$ & $172 \pm 10$ & $3.4 \pm 0.7$ \\
AZ91/0.50 wt.\% GNP & $69 \pm 2.5$ & $128 \pm 13$ & $190 \pm 14$ & $2.8 \pm 0.9$ \\
\hline
\end{tabular}



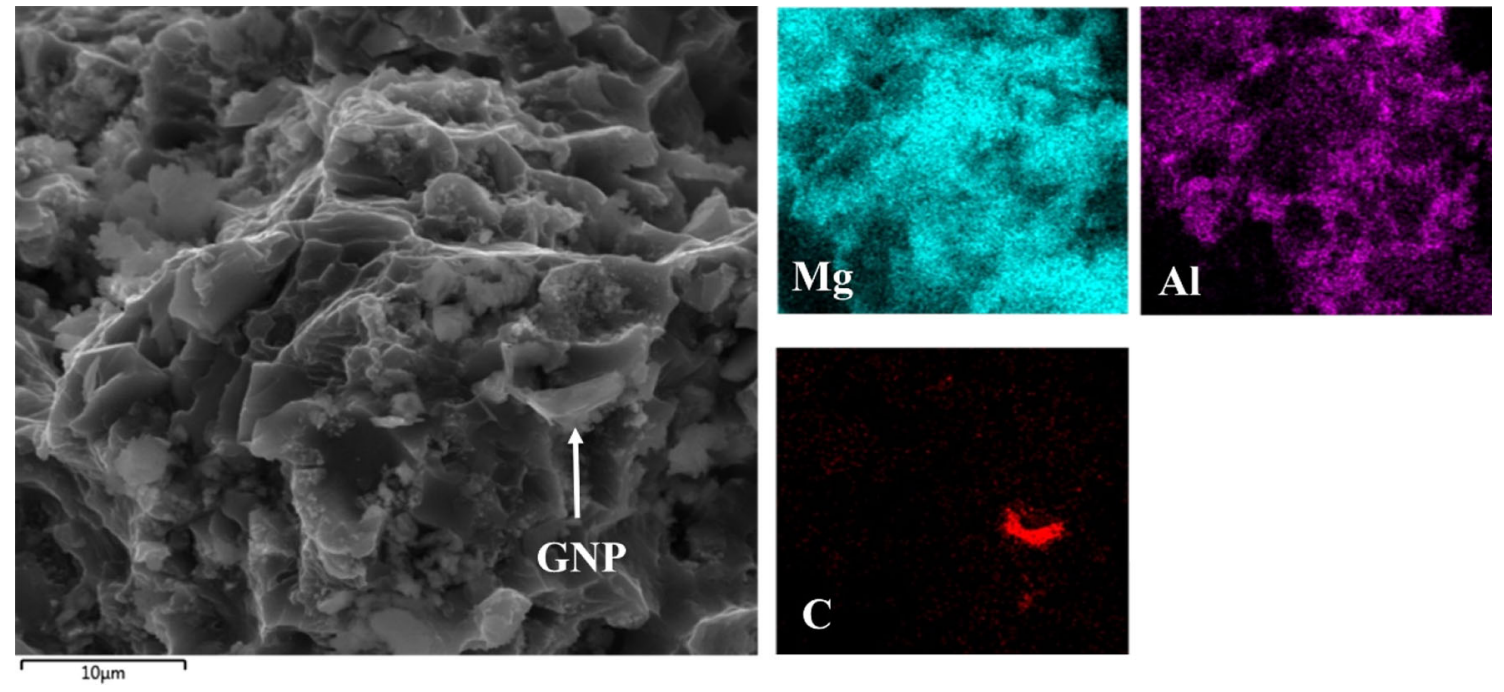

Fig. 10 A representative SEM fracture surface image from the AZ91/0.5 wt.\% GNP composite after tensile testing

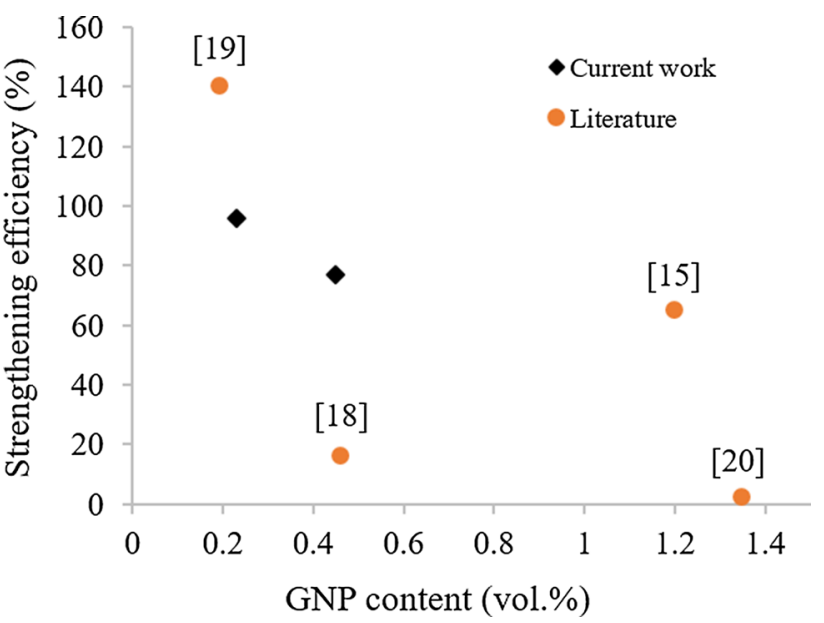

Fig. 11 Comparison of strengthening efficiencies of GNPs in Mgbased metal matrix composites in the current work and the literature (the numbers in the square brackets show the related reference for each set of data)

Unlike widely used globular reinforcements, e.g., nanoparticles, the GNPs have planar structure and coarser sizes (1-2 $\mu \mathrm{m}$ in $x, y$ dimensions, as shown in Fig. 7), and hence larger average interparticle spacing that could induce fewer and weaker barriers to block the dislocation gliding. Therefore, the Orowan strengthening mechanism is expected to have relatively small effect compared to other mechanisms (Ref 28). In addition, the difference between the CTEs of the Mg matrix $\left(26 \times 10^{-6}\left({ }^{\circ} \mathrm{C}\right)^{-1} \quad(\operatorname{Ref} 29)\right)$ and the GNPs $\left(-8 \times 10^{-6}\left({ }^{\circ} \mathrm{C}\right)^{-1}(\operatorname{Ref} 30)\right)$ is more likely to generate plastic strains due to multidirectional thermal stresses and hence dislocation density which leads to strength enhancement. Therefore, along with the small grain sizes, dislocation strengthening mechanism by the CTE mismatch could be suggested to dominate the increments in PS and UTS of composites.

Table 1 also reveals that the elongation to fracture values of GNP-reinforced magnesium matrix composites was significantly higher than that of the AZ91 reference alloy, whereas the addition of GNPs into the matrix could be expected to deteriorate the ductility due to stiffer nature of GNPs. The enhancements in the ductility of composites are most likely to be associated with the transformation of lamellar eutectics to divorced eutectics by the GNP additions (Fig. 6) since the lamellar $\beta$ eutectics which possess large surface area of brittle intermetallic phase are more prone to cracking in comparison with the divorced $\beta$ eutectics (Ref 31 ). It can be also noticed that the elongation of the composite with $0.5 \mathrm{wt} . \%$ GNP was smaller than that of the composite with $0.25 \mathrm{wt} . \%$ GNP and there was only a $10 \%$ increase in the PS and UTS of AZ91/ 0.5 wt. $\%$ GNP composite as compared to the AZ91/0.25 wt.\% GNP composite. This remarkable decline in the elongation and limited strength enhancement may be attributed to relatively high porosity rate and agglomeration tendency of GNPs due to increasing GNP content, i.e., 0.5 wt.\%, as indicated earlier in Fig. 7, since the pores and GNP clusters with reduced fracture strength are potentially preferential sites for premature failure.

Apart from interfacial wetting, content and distribution of GNPs into the matrix, the orientations of GNPs in the composite also play a crucial role in the strengthening due to two-dimensional platelet morphology of GNPs (Ref 17). It can be expected that the strength is further improved when the GNPs are aligned along the tensile direction, and the strength enhancement could be reduced when the GNPs are not aligned along the tensile direction. A representative SEM fracture surface image from the AZ91/0.5 wt.\% GNP composite after tensile testing shows a potential GNP which is perpendicular to the tensile direction and was confirmed by the EDX analysis in Fig. 10 (note that the fracture surface images of all tensile samples are given in Fig. 12, and it was not possible to exactly locate the GNPs aligned along the tensile direction on the fracture surface due to the spatial resolution limit of the EDX system and the fracture surface topography). Therefore, the limited strength enhancement in the composite containing 0.5 wt. $\%$ GNPs may also be attributed to such GNPs embedded into the matrix diminishing the strengthening efficiency.

There are a number of models for prediction of theoretical yield strength enhancement in nanoparticle-reinforced metal matrix composites. Unlike nanoparticles, it is a challenging task to establish a model in order to evaluate the strengthening effect 

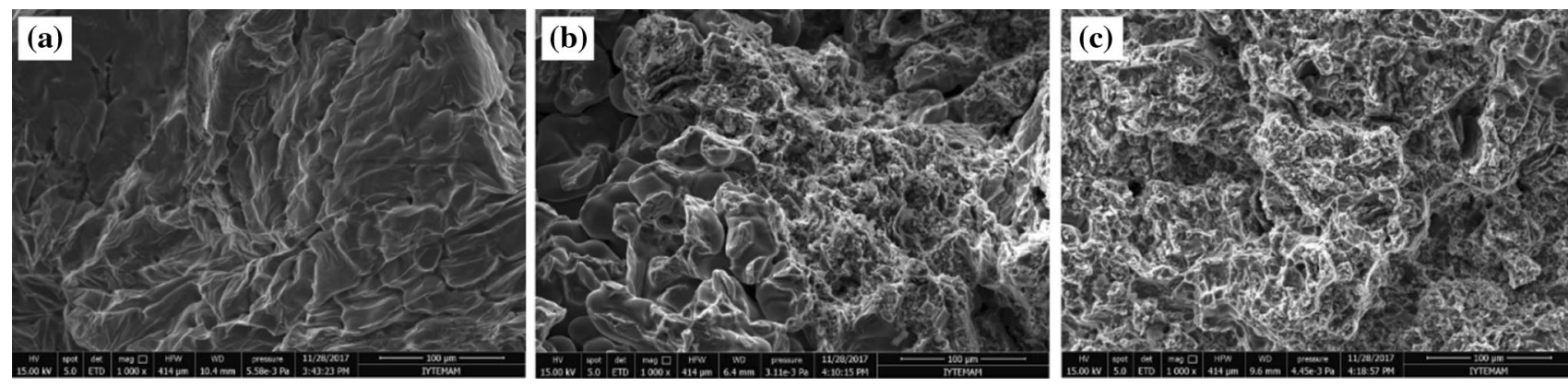

Fig. 12 SEM fracture images of (a) the AZ91 reference alloy, (b) the AZ91/0.25 wt.\% GNP and (c) the AZ91/0.5 wt.\% GNP composites after tensile testing

of randomly distributed GNPs as 2-D nanoscale thin sheets with a large aspect ratio and hence quantitatively analyze their strengthening mechanisms (Ref 15).

The strengthening efficiency (SE), the strengthening effect of a given volume percentage of reinforcement in the matrix, is determined by $\mathrm{SE}=\left(Y_{\mathrm{c}}-Y_{\mathrm{m}}\right) / V_{\mathrm{f}} Y_{\mathrm{m}}$, where $Y_{\mathrm{c}}$ and $Y_{\mathrm{m}}$ are the yield strength of composite and matrix, respectively, and $V_{\mathrm{f}}$ is the volume fraction of reinforcement. The SEs of composites were calculated to be 96 and $77 \%$ for 0.25 and $0.5 \mathrm{wt} . \%$ GNP contents, respectively (note that 0.25 and $0.5 \mathrm{wt} . \%$ GNP contents correspond 0.23 and $0.45 \mathrm{vol} . \%$, respectively). These results may suggest that the composite containing 0.25 wt. $\%$ GNP provides better SE due to more uniform deagglomeration and dispersion of GNPs compared to the composite with 0.5 wt.\% GNP. It was previously reported that GNPs show outstanding strengthening effect on $\mathrm{Mg}$ matrices compared to their counterparts such as CNTs and ceramic-based nanoparticles due to their high strength and large specific surface area leading to larger contact area with the matrix (Ref 15-17). The calculated SEs in the present study were therefore compared with the limited reported data for the GNP-reinforced Mg-based composites fabricated by liquid- or semiliquid-state routes in the literature, as shown in Fig. 11. As can be seen from the graph, the incorporation of GNPs into AZ91 alloys provided significant $\mathrm{SE}$ enhancement. It is noticed that the SE values for both AZ91/0.25 wt.\% GNP and AZ91/0.5 wt.\% GNP are lower than that of Xiang et. al's work (Ref 19). Here, it should be noted that the composites were fabricated by a dynamic solidification process which is a combination of conventional casting and spray processing followed by a hot extrusion process at that work. The extrusion process is considered to be one of the main contributors in obtaining such strengthening. Therefore, the current study shows the potential of solidification processing to produce GNP-reinforced magnesium matrix composites with mechanical properties superior to the unreinforced matrix.

\subsection{Fracture Surface Analysis}

The tensile fracture surfaces of the AZ91 reference alloy and GNP-reinforced AZ91 matrix composites were examined under the SEM, as shown in Fig. 12. The fracture surface of AZ91 alloy without GNP addition exhibited the presence of cleavage steps as observed in Fig. 12(a). On the other hand, as shown in Fig. 12(b) and (c), the fracture surfaces of the composites with 0.25 and 0.5 wt.\% GNP revealed dimples and tear ridges, which can be considered as the signs of ductility enhancement in comparison with the AZ91 reference alloy. Also, some casting defects such as microporosity are visible in Fig. 12(a) and (b).

\section{Conclusions}

In summary, AZ91 magnesium alloy matrix composites with 0.25 and 0.5 wt. $\%$ GNPs were successfully fabricated by a solidification processing which combines mechanical stirring and ultrasonic dispersion of GNPs in the matrix. The following conclusions can be drawn from the experimental findings:

1. The introduction of GNPs into the matrix resulted in a significant reduction in the grain sizes due to the grain boundary pinning effect of the GNPs.

2. The addition of GNPs into AZ91 alloy led to the refinement of $\beta$ eutectics by transforming from lamellar to the divorced eutectics. This morphological alteration was attributed to the fact that the GNPs acted as diffusion barriers and suppressed the diffusion of alloying elements during solidification.

3. The SEM investigations showed that fairly uniform distribution and dispersion of the GNPs into the matrix were achieved few clusters of GNPs observed in the AZ91/ 0.5 wt. $\%$ GNP composite.

4. The TEM analysis suggested that good interfacial bonding was obtained between the matrix and GNPs as no intermediate phase was traced.

5. The mechanical properties of the AZ91 reference alloy were increased with the addition of GNPs. While the enhancements in strength were predominantly attributed to the grain refinement and dislocation generation strengthening by CTE mismatch between the matrix and reinforcement, the improved ductility was attributed to the morphological alteration in the eutectics by the GNPs.

6. The ductility and strengthening efficiency of AZ91/ 0.25 wt. $\%$ GNP composite were higher than those of AZ91/0.5 wt.\% GNP composite. Relatively high porosity rate and agglomeration tendency of GNPs due to increasing GNP content were found to be responsible for those reduced properties. 


\section{Acknowledgments}

This work was supported by "The Scientific and Technical Research Council of Turkey, TÜBITAK” (Grant No. 214M091). The Materials Research Center at Izmir Institute of Technology and the National Nanotechnology Research Center at Bilkent University are acknowledged for the provision of SEM and TEM facilities, respectively. Finally, the author would like to thank Dr. Yunus Türen of Karabük University and Mr. Burak Bostancıoğlu for providing AZ91 ingots and his assistance in carrying out the experiments, respectively.

\section{References}

1. C. Blawert, N. Hort, and K.U. Kainer, Automotive Applications of Magnesium and its Alloys, Trans. Indian Inst. Met., 2004, 57, p 397-408

2. M. Gupta and N.M.L. Sharon, Magnesium, Magnesium Alloys, and Magnesium Composites, Wiley, Hoboken, 2011

3. M. Gupta, M.O. Lai, and D. Saravanaranganathan, Synthesis, Microstructure and Properties Characterization of Disintegrated Melt Deposited Mg/SiC Composites, J. Mater. Sci., 2000, 35, p 2155-2165

4. R.A. Saravanan and M.K. Surappa, Fabrication and Characterization of Pure Magnesium-30 vol.\% $\mathrm{SiC}_{\mathrm{p}}$ Particle Composite, Mater. Sci. Eng. $A, 2000,276$, p 108-116

5. K.B. Nie, X.J. Wang, K. Wu, L. Xu, M.Y. Zheng, and X.S. Hu, Processing, Microstructure and Mechanical Processing of Magnesium Matrix Nanocomposites Fabricated by Semisolid Stirring Assisted Ultrasonic Vibration, J. Alloys Compd., 2011, 509, p 8664-8669

6. M. Gupta and W.L.E. Wong, Magnesium-Based Nanocomposites: Lightweight Materials of the Future, Mater. Charact., 2015, 105, p 30-46

7. L.Y. Chen, J.Q. Xu, H. Choi, M. Pozuelo, X. Ma, S. Bhowmich, J.M. Yang, S. Mathaudhu, and X.C. Li, Processing and Properties of Magnesium Containing a Dense Uniform Dispersion of Nanoparticles, Nature, 2015, 528, p 539-543

8. Q. Li, A. Vierecki, C.A. Rottmair, and R.F. Singer, Improved Processing of Carbon Nanotube/Magnesium Alloy Composites, Compos. Sci. Technol., 2009, 69, p 1193-1199

9. S.C. Tjong, Recent Progress in the Development and Properties of Novel Metal Matrix Nanocomposites Reinforced with Carbon Nanotubes and Graphene Nanosheets, Mater. Sci. Eng. R Rep., 2013, 74, p 281-350

10. M. Paramsothy, X.H. Tan, J. Chan, R. Kwok, and M. Gupta, Carbon Nanotube Addition to Concentrated Magnesium Alloy AZ81: Enhanced Ductility with Occasional Significant Increase in Strength, Mater. Des., 2013, 45, p 15-23

11. C.D. Li, X.J. Wang, K. Wu, W.Q. Liu, S.L. Xiang, C. Ding, X.S. Hu, and M.Y. Zheng, Distribution and Integrity of Carbon Nanotubes in Carbon Nanotube/Magnesium Composites, J. Alloys Compd., 2014, 612, p 330-336

12. V. Singh, D. Joung, L. Zhai, S. Das, S.I. Khondaker, and S. Seal, Graphene Based Materials: Past, Present and Future, Prog. Mater. Sci., 2011, 56, p 1178-1271

13. J. Wang, Z. Li, G. Fan, H. Pan, Z. Chen, and D. Zhang, Reinforcement with Graphene Nanosheets in Aluminum Matrix Composites, Scripta Mater, 2012, 66, p 594-597
14. K. Chu and C. Jia, Enhanced Strength in Bulk Graphene-Copper Composites, Phys. Status Solidi A, 2014, 211, p 184-190

15. L. Chen, H. Konishi, A. Fehrenbacher, C. Ma, J. Xu, H. Choi, H. Xu, F.E. Pfefferkorn, and X. Li, Novel Nanoprocessing Route for Bulk Graphene Nanoplatelets Reinforced Metal Matrix Nanocomposites, Scripta Mater, 2012, 67, p 29-32

16. M. Rashad, F. Pan, A. Tang, M. Asif, J. She, J. Gou, J. Mao, and H. $\mathrm{Hu}$, Development of Magnesium-Graphene Nanoplatelets Composite, J. Compos. Mater, 2015, 49, p 285-293

17. M. Rashad, F. Pan, H. Hu, M. Asif, S. Hussain, and J. She, Enhanced Tensile Properties of Magnesium Composites Reinforced with Graphene Nanoplatelets, Mater. Sci. Eng. A, 2015, 630, p 36-44

18. M. Rashad, F. Pan, and M. Asif, Exploring Mechanical Behavior of Mg-6Zn Alloy Reinforced with Graphene Nanoplatelets, Mater. Sci. Eng. A, 2016, 649, p 263-269

19. S.L. Xiang, M. Gupta, X.J. Wang, L.D. Wang, X.S. Hu, and K. Wu, Enhanced Overall Strength and Ductility of Magnesium Matrix Composites by Low Content of Graphene Nanoplatelets, Compos. Part A, 2017, 100, p 183-193

20. M. Rashad, F. Pan, Y. Liu, X. Chen, H. Lin, R. Pan, M. Asif, and J. She, High Temperature Formability of Graphene Nanoplatelets-AZ31 Composites Fabricated by Stir-Casting Method, J. Magnes. Alloys, 2016, 4, p 270-277

21. A. Srinivasan, J. Swaminathan, M.K. Gunjan, U.T.S. Pillai, and B.C. Pai, Effect of Intermetallic Phases on the Creep Behavior of AZ91 Magnesium Alloy, Mater. Sci. Eng. A, 2010, 527, p 1395-1403

22. A.K. Dahle, Y.C. Lee, M.D. Nave, P.L. Schaffer, and D.H. John, Development of the As-Cast Microstructure in Magnesium-Aluminium Alloy, J. Light Met., 2001, 1, p 61-72

23. S. Candan, M. Unal, E. Koc, Y. Turen, and E. Candan, Effects of Titanium Addition on Mechanical and Corrosion Behaviours of AZ91 Magnesium Alloy, J. Alloys Compd., 2011, 509, p 1958-1963

24. J.Q. Xu, L.Y. Chen, H. Choi, and X.C. Li, Theoretical Study and Pathways for Nanoparticle Capture During Solidification of Metal Melt, J. Phys. Condens. Matter, 2012, 24, p 255304-255314

25. C.D. Li, X.J. Wang, W.Q. Liu, H.L. Shi, C. Ding, X.S. Hu, M.Y. Zheng, and $\mathrm{K}$. Wu, Effect of Solidification on Microstructures and Mechanical Properties of Carbon Nanotubes Reinforced Magnesium Matrix Composite, Mater. Des., 2014, 58, p 204-208

26. Z. Zhang and D. Chen, Consideration of Orowan Strengthening Effect in Particulate-Reinforced Metal Matrix Nanocomposites: A Model for Predicting Their Yield Strength, Scripta Mater., 2006, 54, p 1321-1326

27. L.C. Davis and J.E. Allison, Residual Stresses and Their Effects on Deformation in Particle-Reinforced Metal Matrix Composites, Metall. Trans., 1993, 24, p 2487-2496

28. S.L. Xiang, X.J. Wang, M. Gupta, K. Wu, X.S. Hu, and M.Y. Zheng, Graphene Nanoplatelets Induced Heterogeneous Bimodal Structural Magnesium Matrix Composites with Enhanced Mechanical Properties, Sci. Rep., 2016, 6, p 38824

29. R.E. Taylor, Thermal Expansion of Solids in CINDAS Data Series on Materials Properties, ASM International, Materials Park, 1998, p 1-4

30. D. Yoon, Y.W. Son, and H. Cheong, Negative Thermal Expansion Coefficient of Graphene Measured by Raman Spectroscopy, Nano Lett., 2011, 11, p 3227-3231

31. Y. Turen, Effect of Sn Addition on Microstructure, Mechanical and Casting Properties of AZ91 Alloy, Mater. Des., 2013, 49, p 1009-1015 